Int J Health Care Finance Econ. 2011 September ; 11(3): 165-179. doi:10.1007/s10754-011-9095-5.

\title{
Vertical integration and optimal reimbursement policy
}

\author{
Christopher C. Afendulis and \\ Dapartment of Health Care Policy, Harvard Medical School, Boston, MA, USA \\ Daniel P. Kessler \\ Stanford University Law School, Graduate School of Business, and Hoover Institution, Stanford, \\ CA, USA, The National Bureau of Economic Research, Cambridge, MA, USA \\ Daniel P. Kessler: fkessler@stanford.edu
}

\begin{abstract}
Health care providers may vertically integrate not only to facilitate coordination of care, but also for strategic reasons that may not be in patients' best interests. Optimal Medicare reimbursement policy depends upon the extent to which each of these explanations is correct. To investigate, we compare the consequences of the 1997 adoption of prospective payment for skilled nursing facilities (SNF PPS) in geographic areas with high versus low levels of hospital/SNF integration. We find that SNF PPS decreased spending more in high integration areas, with no measurable consequences for patient health outcomes. Our findings suggest that integrated providers should face higher-powered reimbursement incentives, i.e., less cost-sharing. More generally, we conclude that purchasers of health services (and other services subject to agency problems) should consider the organizational form of their suppliers when choosing a reimbursement mechanism.
\end{abstract}

\section{Keywords}

Health economics; Industrial organization; Reimbursement policy

\section{Introduction}

Over the past 20 years, markets for health services have become significantly more vertically integrated. Producers of complementary services that were once independent are now increasingly commonly-owned. By the end of the 1990s, three-quarters of acute-care hospitals had acquired a supplier of post-acute care such as a rehabilitation hospital, home health service, or skilled nursing facility (SNF) (U.S. Congressional Budget Office 1997). The most important of these from the perspective of the Medicare program are SNFs, which account for the largest share of post-acute spending (Medicare Payment Advisory Commission 2010).

In theory, the optimal response of Medicare to the integration of hospitals into post-acute services is indeterminate. Because providers have better information on the severity of patients' illness, optimal reimbursement policy generally allows them to pass on some but not all of the costs of treatment. This well-known result reflects the tradeoff between incentives not to skimp on treatment and incentives to contain costs (Ellis 1998). Because integration may change the terms of this tradeoff in either direction, or not at all, whether

(C) Springer Science+Business Media, LLC 2011

Correspondence to: Daniel P. Kessler, fkessler@stanford.edu. 
integrated post-acute care providers should be offered more or less cost-sharing than their stand-alone counterparts is an empirical question.

On one hand, integration may enhance these providers' incentives to supply unnecessary treatment. This could occur, for example, if integration is used as a vehicle to pay kickbacks for inefficient referrals (Pauly 1979). ${ }^{1}$ Inefficient referrals for post-acute care are of particular interest in the Medicare program, because acute-care hospitals have traditionally faced higher-power reimbursement than post-acute care providers. ${ }^{2}$ This anomaly could give integrated hospitals greater incentives to discharge patients to post-acute facilities prematurely, thereby resulting in more avoidable post-acute stays (e.g., Morrisey et al. 1988). In this case, giving integrated providers more powerful incentives for cost containment could limit spending and improve efficiency.

On the other hand, integration may enhance post-acute providers' incentives to skimp. This could occur, for example, if integration inhibits Medicare's ability to monitor quality of care, or if integration enables providers to better align the incentives of physician decisionmakers with their own at patients' expense. In this case, giving integrated providers less powerful incentives for cost containment could be optimal.

Integration may also have no effect on the principal/agent relationship between Medicare and post-acute care providers, if (for example) it is simply a way to facilitate coordination of care. In this case, integration would not change incentives for skimping or cost containment, and should therefore not be a factor in determining the optimal cost-sharing rule.

Despite the importance of this question, little research has examined the relationship between hospital integration and Medicare reimbursement policy. In this article, we evaluate the effects of a change in such policy on health spending and the quality of care. In 1997, Congress adopted prospective payment for skilled nursing facilities (SNF PPS), under which SNFs are paid a largely fixed amount per patient per day based on the patient's anticipated resource use. ${ }^{3}$ Prior to this change, Medicare reimbursed SNFs on the basis of their costs. This change therefore led to decreased cost-sharing for both stand-alone SNFs and those that were integrated with an acute-care hospital. ${ }^{4}$

We analyze the Medicare spending and health outcomes of all elderly patients admitted to an acute-care hospital with stroke in 1997, 1998, and 1999. Rehabilitation services provided by SNFs are frequently, but not always, indicated for patients who are hospitalized with this disease (Gropen et al. 2009).

We compare the pre/post-SNF-PPS change in the spending on and the health outcomes of an episode of illness in geographic areas with a high versus a low level of hospital/SNF integration. We hold constant the characteristics of patients, hospitals, geographic markets, and other aspects of Medicare reimbursement policy that were changing over our study period, including area-level measures of the generosity of Medicare for both hospital and SNF services. This enables us to assess whether enhanced incentives for cost containment have different effects on spending and health outcomes, depending on the extent of vertical integration.

\footnotetext{
${ }^{1}$ Explicit payments for referrals are banned by Medicare (and Medicaid), effectively eliminating them, but the legal prohibition can be circumvented by integration, because policing transfer payments among parties that share fixed assets within an organization is extremely difficult.

${ }^{2}$ Before 1997, acute-care hospitals were reimbursed under a prospective payment system and post-acute facilities under a cost-plus system, which creates an opportunity for profit from shifting care inappropriately from the acute to the post-acute setting.

${ }^{3}$ Congress later adopted prospective payment for other forms of post-acute care.

${ }^{4}$ We describe SNF PPS as leading to decreased cost-sharing, rather than no cost-sharing, because it still allowed for cost-plus reimbursement on some margins such as the number of days in the SNF and certain therapy services (White 2005/2006).
} 
The article proceeds in five sections. The "Previous literature" section outlines the previous research on the role of vertical integration in markets for health care, with a focus on strategic uses of integration in a principal/agent context. The "Data" and "Model" sections present our data and models. Results section presents our results, and the "Conclusion" section concludes with some suggestions for Medicare policy and contracting more generally.

\section{Previous literature}

Conventional models of the boundaries of the firm explain how vertical integration in markets for health services can enhance efficiency. Common ownership of each part of the "supply chain" involved in caring for patients who need hospital services, for example, can facilitate coordination of care, address incomplete contracting problems, and otherwise reduce transaction costs (Robinson 1996).

More recent models explore how vertical integration can inhibit competition. Several papers demonstrate how vertical relationships can confer market power if there are barriers to entry (e.g., Riordan and Salop 1995; Bernheim and Whinston 1998). Galor (1999) applies these concepts to a model of bargaining between hospitals, physicians, and managed care plans. Empirical papers reach opposing conclusions about the applicability of these models to markets for hospital services. Using data from Arizona, Florida, and Wisconsin for 19941998, Cuellar and Gertler (2006) examine whether hospitals that are integrated with their physicians have different accounting costs per patient, adjusted charges per patient, and quality of care than hospitals that are not. They find no systematic effects of integration on costs or quality of care, but a positive effect of integration on charges. However, using data from California for 1994-2001, Ciliberto and Dranove (2006) find no systematic effects of integration on charges per patient.

Fewer papers model how vertical integration affects the principal/agent relationship between providers and insurers and the associated tradeoff between cost containment and skimping that is at the heart of reimbursement policy design. Pauly (1979) explains one reason why this may be important: integration allows providers, at least implicitly, to pay one another for referrals. Providers sell not only treatments themselves, but also advice about what treatments to obtain. Because providers may not act as perfect agents for patients or their insurers, allowing providers to make and receive payments for referrals may lead to medically unnecessary health spending (e.g., Afendulis and Kessler 2007). To the extent that this occurs, giving integrated providers more powerful incentives for cost containment could limit spending and improve efficiency. 5

Vertical integration may affect the principal/agent relationship in other ways. Integration may make it more difficult for Medicare to monitor the quality of service provided by individual entities, or may enhance the ability of hospitals and/or post-acute care facilities to manage their physicians for the facilities' financial benefit. These sorts of effects are consistent with models such as Laffont and Martimort (1997) and Baron and Besanko (1992), which show how vertical integration can be used to alter the information structure in a more general principal/agent context. To the extent that this occurs, giving integrated providers less powerful incentives for cost containment could be optimal.

\footnotetext{
${ }^{5}$ The canonical concern is that payments for referrals may induce providers to recommend more profitable treatments even if they are not in patients' interest. However, even if payments for referrals induce referring providers to give more efficient advice (by allowing them to capture gains from referring patients who would be better served by someone else), it might still be optimal to offer integrated providers more powerful incentives for cost containment, if such incentives encourage providers to improve their division of labor (e.g., Garicano and Santos 2004).
} 
One recent empirical paper suggests that integration by hospitals may be facilitating payments for referrals. Using data from Florida and New York from 1994-2002, Nakamura et al. (2007) find that large tertiary care hospitals that acquire community hospitals increase their referrals, particularly among patients undergoing more profitable procedures and with more generous insurance. However, although this work is informative, it can neither reject conventional transaction-cost explanations for integration nor provide guidance to purchasers and policy-makers about their optimal response to providers' decisions about organizational form.

In this article, we seek to fill this gap. We evaluate the consequences of SNF PPS by examining the spending on and the health outcomes of elderly Medicare beneficiaries admitted to an acute-care hospital with stroke. Our research design is essentially a difference-in-difference (DD) framework: we compare spending before versus after SNF PPS in geographic areas with a high versus a low level of SNF/hospital integration. We examine not only spending overall, but also spending on SNF care and its substitutesrehabilitation hospital, outpatient, and home health spending (Gresham 1997). We also examine an extensive set of patient health outcomes, including several measures found by previous research to be specifically related to the quality of SNF care. Finally, to isolate the effect of the interaction between payment incentives and vertical integration, we control for an extensive set of patient, market, and reimbursement policy characteristics.

Our article also contributes to the health services literature on the effects of reimbursement incentives on cost and quality in markets for health care. Most of this work (e.g., DesHarnais 1987; Newhouse and Byrne 1988; Ellis and McGuire 1990; Cutler 1995; McClellan 1997) has focused on Medicare's 1983 adoption of a higher-powered payment system for acutecare hospitals. Fewer papers have examined the effects of SNF PPS. Banks et al. (2001) present a theoretical model that highlights the conditions under which SNF PPS will induce hospitals and SNFs to combine their services efficiently. Konetzka et al. (2006) find that the shift to SNF PPS, holding constant the average level of reimbursement, increased the rate of adverse health outcomes for SNF patients. White (2005/2006) suggests that this result is due, at least in part, to declines in nurse staffing in response to the change in reimbursement policy. In contrast, Wodchis et al. (2004/2005) find that SNF PPS reduced mortality for Medicare beneficiaries admitted to a SNF and had no significant effect on rehospitalizations.

\section{Data}

We analyze the spending and health outcomes of all Medicare patients 65 years or older who were admitted to an acute-care hospital in the years 1997, 1998, and 1999 with stroke.

Because BBA 1997 and SNF PPS were partially implemented in 1998 and fully implemented by 1999, we can estimate the partial and full consequences of the policies with our study period.

We examine patients with a principal diagnosis of occlusive stroke with infarction (ICD-9CM 433.x1 or 434.x1) or transient cerebral ischemia (ICD-9-CM 435). We found 672,973 such strokes for 1997-1999. To ensure that we have complete claims records for all patients, we restrict our sample to patients who were enrolled in fee-for-service Medicare continuously for the 365 days preceding and the 365 days following their initial admission in each calendar year. We also exclude beneficiaries admitted for a stroke in the 12 months prior to the index event and beneficiaries residing outside the United States. These exclusion restrictions left us with 507,350 observations. ${ }^{6}$

\footnotetext{
${ }^{6}$ Our analyses of total and home health spending are based on a $20 \%$ random sample of beneficiaries, because home health spending (and therefore total spending) is only available for this subsample. All other analyses are based on the 100\% sample of 507,350.
} 
We assess the consequences of integration for the following financial and health outcomes $Y$ : total spending (including all inpatient and outpatient hospital care, and patient out-ofpocket spending and Medicare payments on the patient's behalf); whether the patient received treatment from a SNF, a rehabilitation hospital, an outpatient hospital, and a home health agency; the amount of spending on SNF, rehabilitation hospital, outpatient hospital, and home health agency care; whether the patient was readmitted to an acute-care hospital in the 31-365 days after admission ${ }^{7}$; and 365-day all-cause mortality. In addition, we also used the primary and secondary diagnosis fields in each stay in the 31-365 days after the index event to code six rehospitalization measures used to measure quality of care for patients admitted to a SNF: congestive heart failure, anemia, electrolyte imbalance, respiratory infection, sepsis, and urinary tract infection. ${ }^{8}$

To isolate the effect of the interaction between payment incentives and vertical integration, we control for an extensive set of patient, market, and reimbursement policy characteristics. Patient characteristics include: age (66-69, 70-74, 75-79, 80-84, 85 or older), gender, and race; the 30 risk-adjustment variables described by Elixhauser et al. $(1998)^{9}$; and total Medicare inpatient spending in the 365 days prior to the index event (reflecting both patient out-of-pocket spending and Medicare payments on the patient's behalf).

We calculate several characteristics of the health care market in the patient's geographic area in 1997, which we define as the 3-digit Zip Code around the patient's residence ( $K=$ 860 ). We calculate the percentage of SNF beds integrated with a hospital, $V$, in the patient's area in 1997, before the change in Medicare reimbursement policy. In addition, we calculate the following other characteristics $Z$ in 1997: the percentage of hospital beds by ownership status (for-profit, non-profit, government-owned), facility size (100 beds or fewer, 101-500, more than 500), teaching status, and system membership; the percentage of SNF beds by ownership status and facility size ( 25 or fewer, 26-50, more than 50); the number of SNF beds per population aged 66 or older; and Herfindahl-Hirschman indices of hospital and SNF competition, as measured by the number of hospital and SNF beds, respectively. Each of these characteristics in $V$ and $Z$ is coded as 1 if the area level of the variable was above the nationwide hospital-bed-weighted median for each underlying measure, 0 otherwise.

Finally, we calculate area-level measures of the generosity of Medicare reimbursement for both hospital and SNF services $G$ for each year from 1997-1999. Controlling for generosity is important in assessing the effects of the shift to higher-powered payment incentives in BBA 1997. Several researchers (e.g., White 2005/2006) have pointed out that BBA 1997 affected both the level of reimbursement on average as well its responsiveness to costs, and both of these parameters may affect the cost and quality of care. The details behind the calculation of $G$ are described in Appendix A.

Table 1 presents descriptive statistics for the population of elderly beneficiaries that we analyze for 1997 and 1999, and previews the results of our analysis. The top panel of the table presents descriptive statistics on spending in the year after admission for stroke; the second panel, on health outcomes; and the third panel, on spending in the year prior to the index event. The table reports the expenditures, background characteristics, and health outcomes of patients in two types of areas-areas with a high level of integration between hospitals and SNFs $(V=1)$ and areas with a low level of integration $(V=0)$.

\footnotetext{
${ }^{7}$ Optimal follow-up care is designed to prevent readmissions to the hospital if possible; we exclude readmissions in the month after initial admission because these may be part of the initial course of care. Mor et al. (2010) show that rehospitalizations after a SNF stay are an important measure of the quality of care for episodes of illness requiring post-acute services

${ }^{8}$ See Kramer et al. (2007).

${ }^{9}$ These variables were coded using the DRG and secondary diagnosis codes for all stays in the 365 days prior to the index event.
} 
The first row of the top panel shows that total spending declined nationwide for this population from 1997-1999, but that it declined more for patients living in areas with a high level of hospital/SNF integration. In the 365 days following admission, spending in moreintegrated areas declined by $11 \%$, whereas spending in less-integrated areas declined by $6.1 \%$; SNF PPS thus reduced spending in integrated areas by an additional $4.9 \%$. The differential decline was particularly pronounced for spending on SNF services, where SNF PPS should have had the greatest effect. In addition, there were no offsetting increases on spending on other forms of post-acute care that might serve as a substitute for SNF services, such as rehabilitation hospitals, outpatient facilities, or home health.

The second panel shows that there are no major trends in health outcomes for stroke over our study period, in either in absolute terms or differentially across areas. A substantial share of patients were readmitted to the hospital (approximately 42\%), but the pre-/post-PPS difference was at most 0.2 percentage points, or one-half of $1 \%$. Among the six outcomes highlighted by Kramer et al. (2007) as being specific measures of the quality of SNF care, the differential trends in outcomes are even smaller. Mortality in high integration areas increased more than mortality in low integration areas ( 0.2 percentage points), but this too amounts to a relatively small change on a base of approximately 16 percentage points.

The third panel presents patients' average spending on acute-care hospital services in the year prior to the index event, as a summary measure of patients' health status on admission. Although this measure is much coarser than the detailed controls for health status that we ultimately use in estimation, it suggests that the differences in trends in spending reported in the top panel are not due to differences patient health status between high and low integration areas. If anything, patients in high integration areas had slightly greater growth in their year-prior-to-index-event hospital spending $(0.9 \%$ as compared to $-0.1 \%)$.

The simple differences in trends in spending in the year after onset of illness, along with a lack of difference in outcome trends, suggest that increasing incentives for cost containment is an optimal response to vertical integration between hospitals and SNFs. When Medicare shifted to a higher-powered reimbursement scheme for SNFs, SNF spending on patients from high integration areas declined more than spending on patients from low integration areas. The trends in spending were not accompanied by any trend in health outcomes, suggesting that the medical productivity of the eliminated services were low. It also suggests that such integration is a strategic decision to facilitate payment for referrals. If integration were solely a means to reduce transaction costs, there would be no reason to expect SNF PPS to have different effects in high versus low integration areas. Of course, these conclusions are only speculative, since the descriptive statistics presented above do not comprehensively control for the characteristics of patients, hospital and SNF market areas, and other changes in reimbursement policy that occurred over our study period. In the following section, we formalize the intuition behind these differences and state the assumptions that are necessary to test our hypothesis.

\section{Model}

In each 3-digit Zip Code $k=1, \ldots, K$ during $t=1997,1998,1999$, we examine the spending on and quality of care of each elderly fee-for-service Medicare beneficiary $i=1, \ldots, N_{k t}$ who was admitted to an acute-care hospital with stroke. We estimate how the spending on treatment and health outcomes of each patient, $Y_{i k t}$, differed in areas with and without significant vertical integration between hospitals and SNFs, $V_{k}$. We also estimate how this difference changed contemporaneous with adoption of SNF PPS. In addition to each patient's financial and health outcomes, we also observe each patient's personal 
characteristics $X_{i k t}$, hospital and SNF market characteristics $Z_{k}$, and generosity of Medicare reimbursement for acute-care and SNF services $G_{k t}$.

Our regression models specify $Y_{i k t}$ as a function of time fixed effects $\theta_{t}, X_{i k t}, G_{k t}, Z_{k}$, and $V_{k}$; interactions between $G_{k t}$ and $V_{k}$; interactions between $\theta_{t}$ and $Z_{k}, V_{k}$; and an error term $\varepsilon_{i k t}{ }^{10}$

$$
Y_{i k t}=\theta_{t}+X_{i k t} \beta+G_{k t} \gamma+Z_{k} \varphi+V_{k} \delta+\left(G_{k t} \times V_{k}\right) \gamma^{V}+Z_{k} \theta_{t}^{Z}+V_{k} \theta_{t}^{V}+\varepsilon_{i k t} .
$$

In this model, the coefficient of interest is $\theta_{t}^{V}$, the differential 1997-1999 growth rate of medical spending or health outcomes in a geographic area with a high versus a low density of vertical integration.

Interpreting estimates of $\theta_{t}^{V}$ as evidence of an interaction between more powerful incentives for cost containment and vertical integration requires three important assumptions. First, it requires the assumption that the residual difference in 1997-1999 trends between high and low integration areas is attributable to SNF PPS. Over our brief study period, SNF PPS was the only important change to the market for skilled nursing care, and SNF PPS had two effects: to decrease the responsiveness of reimbursement to costs, and to decrease the overall level of reimbursement. We control for $G_{k t}$ and the interactions between $G_{k t}$ and $V_{k}$ to make this assumption as plausible as possible. Although it is certainly possible that other, longerterm changes to health care markets were occurring at this time, we do not know of any that we would expect to have affected SNF spending on elderly Medicare beneficiaries differently in integrated and nonintegrated areas. Second, it requires the assumption that we have modeled all of the other differences across areas that might have mediated the effects of the changes in reimbursement policy imposed by BBA 1997. We control for the interactions of other hospital market characteristics $Z_{k}$ with $\theta_{t}$ to make this assumption as plausible as possible. Third, it requires the assumption that there are no differential changes across areas in the unobserved characteristics or health status of patients. We control for the demographic characteristics and diagnoses of patients at their index admission, and for their diagnoses and inpatient spending in the year prior to admission, to make this assumption as plausible as possible.

\section{Results}

Table 2 presents estimates of $\theta_{t}, \delta$ and $\theta_{t}^{V}$ from Eq. 1, using various measures of health spending as the dependent variable $Y_{i k t}$. The reported standard errors allow for arbitrary clustering of $\varepsilon_{i k t}$ within a 3-digit Zip Code $k$. Column 1 of the table contains the article's key results. The first two rows present estimates of $\theta_{t}$, which capture the baseline trend in spending, holding constant individual, market, and reimbursement policy characteristics. These estimates are statistically insignificant, which suggests that our controls for $G_{k t}$ are capturing the decreasing generosity of reimbursement of SNF PPS that other researchers

\footnotetext{
${ }^{10}$ For two reasons, we model $Y_{i k t}$ as a function of 1997 area-level measures of hospital characteristics $Z_{k}$ and $V_{k}$, rather than contemporaneous individual-level measures $Z_{i k t}$ and $V_{i k t}$. We use area-level rather than individual-level measures to eliminate omitted variable bias due to patient selection of hospitals. Omitted variable bias arises in this context because patients choose hospitals on the basis of their health status; health status is not fully observable; and the unobservable dimensions of health also affect skillednursing spending. As a result, OLS estimates of the effect of individual patients' hospital characteristics are in general inconsistent. (Consistent estimates of the effect of hospital characteristics can be obtained using area-level measures as instruments, but this approach requires the additional assumption that area-level measures have no effect on spending, except through their impact on patients' hospital choice, which may be incorrect.) We use 1997 (baseline) measures instead of contemporaneous measures to eliminate simultaneity bias due to hospital responses to SNF PPS. Simultaneity bias arises in this context because hospitals may seek to change their organizational form in response to changes in Medicare reimbursement policy. As a result, OLS estimates of the effect of contemporaneous measures of hospital markets may be inconsistent.
} 
have documented. The level of overall spending in high integration areas also does not differ significantly from that in low integration areas. However, by 1999, the payment reforms in BBA 1997 had phased in for most providers and transformed Medicare reimbursement for SNF care. Concurrent with this was a statistically significant negative integrated-area spending differential, $\theta_{t}^{V}$, of $5.2 \%$ (standard error 2.2).

As the table indicates, this estimate was obtained on a $20 \%$ random sample of 101,137 patients rather than on the full population of 505,076 patients because home health spending data are only available for the $200 \%$ random sample. To verify that our finding is not due to an anomaly of the $20 \%$ sample, we also estimated the model on the $100 \%$ sample, using as the dependent variable total spending net of home health. Results from this model are virtually the same. The baseline trend in spending was statistically insignificantly negative; the level of overall spending in high integration areas does not differ significantly from that in low integration areas; and the estimate of $\theta_{t}^{V}$ was $-4.1 \%$ (standard error 1.6).

The remaining columns of Table 2 report estimates of $\theta_{t}, \delta$, and $\theta_{t}^{V}$ on selected components of spending. Column 2 shows that the probability of any SNF spending declined by 1.2 percentage points more in integrated areas; the column 3 shows that the level of SNF spending, conditional on a SNF admission, also declined, by 5.3\%. Columns 4-7 show that the decline in SNF utilization was accompanied by some offsetting increases in rehabilitation and outpatient utilization. Patients in more-integrated areas were 1.2 percentage points more likely to be admitted to a rehabilitation hospital (although, conditional on admission, spent $7.4 \%$ less (columns 4-5), and 1.1 percentage points more likely to have an outpatient visit (although only marginally statistically significant, column 6). There is no evidence of offsetting spending on home health; in fact, patients in high integration areas had $8.1 \%$ slower growth in home health spending (although only marginally statistically significant, column 9).

Table 3 presents estimates of $\theta_{t}, \delta$, and $\theta_{t}^{V}$ from Eq. 1, using various measures of health outcomes as the dependent variable. The table shows that there is no evidence of adverse effects on health outcomes of the increasing incentives for cost containment associated with SNF PPS. For no measure is the baseline 1997-1999 trend positive and statistically significant; for mortality, the baseline trend is negative and marginally statistically significant, consistent with Wodchis et al. (2004/2005). There is also no evidence of a greater incidence of adverse outcomes in high versus low integration areas. For no measure is the difference in trend in adverse outcomes statistically significant, either positive or negative.

Regardless of the statistical significance of the interaction between PPS and integration on outcomes, differences in trends in outcomes between high and low integration areas are very small and precisely estimated. Estimated coefficients on $\theta_{t}^{V}$ are at most two-tenths of one percentage point with standard errors of approximately the same magnitude. This underscores that the additional services supplied in high integration areas before BBA 1997 had very minimal benefits for patient health.

\section{Conclusion}

Typically, economic models assume that vertical integration is either driven by a desire to minimize transaction costs or a desire to foreclose competition. In markets for health services, sellers may have another reason to integrate: to enable them to pay one another for referrals. Payments for referrals are banned for Medicare and Medicaid patients, and either banned or highly restricted for patients covered by private insurance. However, if the 
integrating parties share fixed assets, then common ownership can circumvent these controls through creative cost accounting.

If vertical integration is used to make payments for referrals, its implications for optimal reimbursement policy are theoretically ambiguous. If integration enhances incentives to supply unnecessary treatment, then giving integrated providers higher-powered incentives for cost containment could reduce health spending without harming patient health outcomes. But if integration enhances incentives for skimping on care, then optimal policy should offer integrated providers greater opportunities for cost sharing.

In this article, we investigate the extent to which the implementation of a higher-powered, prospective payment system for skilled nursing care (SNF PPS) affected the consequences of integration between hospitals and skilled nursing facilities. We examine the health spending and health outcomes of elderly patients admitted to the hospital with a stroke, an illness for which skilled nursing care is sometimes (although not always) medically indicated. Our research design is essentially a difference-in-difference framework that compares areas with high versus low levels of integration between hospitals and SNFs before versus after Medicare adopted SNF PPS, holding the other characteristics of areas constant.

We find that SNF PPS reduced spending more in high integration areas. Holding constant the generosity of reimbursement, other market characteristics, and the characteristics of patients, integrated areas showed greater declines in SNF spending when Medicare shifted to a higher-powered reimbursement mechanism. Declines in spending were statistically significant and economically important, approximately $5 \%$ of the total costs of care for patients with stroke, or $\$ 1,000$. There is no evidence that these declines were due to changes in the observable characteristics of patients, and no evidence that they were accompanied by changes in health outcomes. There is also no evidence that SNF PPS harmed patients in low integration areas. Our findings therefore suggest that integrated providers should face higher-powered reimbursement incentives, i.e., less cost-sharing, than their stand-alone counterparts. Given that there are approximately 170,000 elderly beneficiaries with this illness admitted to the hospital every year, we estimate that SNF PPS saved \$170 million in this population alone.

Our analysis has several limitations. Most significantly, it depends on the assumption that the residual difference in 1997-1999 trends between integrated and non-integrated areas is attributable to Medicare's shift to higher-powered payment incentives. To make this assumption as plausible as possible, we hold constant detailed patient characteristics, several variables measuring market conditions, and the generosity of payment. The fact that our regression estimates are very close to and not statistically distinguishable from simple differences in mean trends gives us confidence in the validity of this assumption, because unobservables are generally correlated with observables. In addition, our finding of no effect on health outcomes should be interpreted cautiously because of the coarseness of our measures. For example, our analysis fails to account for benefits such as faster or more complete rehabilitation, to the extent they are not captured by claims data; for patients with stroke, these benefits may be important.

Yet, our findings do suggest that purchasers of health services (or other services subject to agency problems) should consider the organizational form of their suppliers when choosing a reimbursement mechanism. Even if integration among suppliers of complementary inputs does reduce transaction costs, it may be second-best optimal for a purchaser to offer integrated suppliers a higher-powered payment regime. Future research might investigate more generally the extent to which incentives in reimbursement and contracting can be used 
to induce agents with private information to choose their organizational form to enhance overall efficiency.

\section{Acknowledgments}

We gratefully acknowledge funding from the National Institutes on Aging through the NBER. Afendulis also gratefully acknowledges funding from the Marshall J. Seidman Program in Health Economics in the Department of Health Care Policy at Harvard Medical School.

\section{References}

Afendulis C, Kessler D. Tradeoffs from integrating diagnosis and treatment in markets for health care. American Economic Review. 2007; 97:1013-1020.

Banks D, Parker E, Wendel J. Strategic interaction among hospitals and nursing facilities: The efficiency effects of payment systems and vertical integration. Health Economics. 2001; 10:119134. [PubMed: 11252043]

Baron DP, Besanko D. Information, control, and organizational structure. Journal of Economics and Management Strategy. 1992; 1:237-275.

Bernheim BD, Whinston MD. Exclusive dealing. Journal of Political Economy. 1998; 106(1):64-103.

Ciliberto F, Dranove D. The effect of physician-hospital affiliations on hospital prices in California. Journal of Health Economics. 2006; 25:29-38. [PubMed: 16352360]

Cuellar A, Gertler PJ. Strategic integration of hospitals and physicians. Journal of Health Economics. 2006; 25:1-28. [PubMed: 16309766]

Cutler DM. The incidence of adverse medical outcomes under prospective payment. Econometrica. 1995; 63:29-50.

DesHarnais S. The early effects of the prospective payment system on inpatient utilization and the quality of care. Inquiry. 1987; 24:7-16. [PubMed: 2951337]

Elixhauser A, Steiner C, Harris DR, Coffey RM. Comorbidity measures for use with administrative data. Medical Care. 1998; 36:8-27. [PubMed: 9431328]

Ellis RP. Creaming, skimping, and dumping: Provider competition on the intensive and extensive margins. Journal of Health Economics. 1998; 17:537-555. [PubMed: 10185511]

Ellis RP, McGuire TG. Optimal payment systems for health services. Journal of Health Economics. 1990; 9:375-396. [PubMed: 10109988]

Galor E. The profitability of vertical mergers between hospitals and physician groups. Journal of Health Economics. 1999; 18:623-654. [PubMed: 10621368]

Garicano L, Santos T. Referrals. American Economic Review. 2004; 94:499-525.

Gresham GE. Rehabilitation. Stroke. 1997; 28:1522-1527. [PubMed: 9227710]

Gropen T, Magdon-Ismail Z, Day D, Melluzzo S, Lee H Schwamm. on behalf of the NECC Advisory Group. Regional implementation of the stroke systems of care model: Recommendations of the northeast cerebrovascular consortium. Stroke. 2009; 40:1793-1802. [PubMed: 19299641]

Konetzka RT, Norton EC, Stearns SC. Medicare payment changes and nursing home quality: Effects on long-stay residents. International Journal of Health Care Finance and Economics. 2006; 6:173189. [PubMed: 17016764]

Kramer, A.; Eilertsen, T.; Goodrich, G.; Min, S. [Accessed 8 August 2011] Understanding temporal changes in and factors associated with SNF rates, MedPAC. 2007. Available at http://www.medpac.gov/documents/Jun07_SNF_contractor_CC.pdf

Laffont JJ, Martimort D. Collusion under asymmetric information. Econometrica. 1997; 65:875-911.

McClellan MM. Hospital reimbursement incentives: An empirical approach. Journal of Economics and Management Strategy. 1997; 6:91-128.

Medicare Payment Advisory Commission. [Accessed 11 July 2011] Post-acute care databook. 2010. http://www.medpac.gov/chapters/Jun10DataBookSec9.pdf

Mor V, Intrator O, Feng Z, Grabowski DC. The revolving door of rehospitalization from skilled nursing facilities. Health Affairs. 2010; 29:57-64. [PubMed: 20048361] 
Morrisey MA, Sloan FA, Valvona J. Shifting medicare patients out of the hospital. Health Affairs. 1988; 5:52-64. [PubMed: 3068108]

Nakamura S, Capps C, Dranove D. Patient admission patterns and acquisitions of "feeder" hospitals. Journal of Economics and Management Strategy. 2007; 16(4):995-1030.

Newhouse JP, Byrne DJ. Did medicare's prospective payment system cause length of stay to fall? Journal of Health Economics. 1988; 7:413-416. [PubMed: 10303151]

Pauly MV. The ethics and economics of kickbacks and fee-splitting. RAND Journal of Economics. 1979; 10(1):344-352.

Riordan MH, Salop S. Evaluating vertical mergers: A post-Chicago approach. Antitrust Law Journal. 1995; 63:513-568.

Robinson JC. Administered pricing and vertical integration in the hospital industry. Journal of Law and Economics. 1996; 39:257-278.

U.S. Congressional Budget Office. [Accessed 8 August 2011] Medicare spending on post-acute care services: A preliminary analysis. 1997. Available at http://www.cbo.gov/ftpdocs/106xx/doc10605/1997-01-acute.pdf

White C. Medicare's prospective payment system for skilled nursing facilities: Effects on staffing and quality of care. Inquiry. 2005/2006; 43:351-366.

Wodchis WP, Fries BE, Hirth RA. /2005) The effect of medicare's prospective payment system on discharge outcomes of skilled nursing facility residents. Inquiry. 2004; 41:418-434. [PubMed: 15835600]

\section{Appendix A: Construction of generosity measures}

For each ZIP3, we calculated measures of Medicare payment generosity for hospitals and SNFs.

For hospital generosity, we started with claims data for all PPS hospital stays $j=1, \ldots, J$ from $t=1997,1998,1999$ in zip codes $k=1, \ldots, K$. We used charge data from these claims records and cost-to-charge ratios from the hospital cost reports to construct a stay-level measure of costs, $C_{j k t}$. We also constructed a stay-level measure of expenditures, $E_{j k t}$. We then ran regressions separately for each year of $C_{j k t}$ and $E_{j k t}$ on a vector of controls $X_{j k t}$, including a Black/non-Black indicator, a female/male indicator, a measure of hospital spending in the year prior to admission, age dummies $(66-69,70-74,75-79,80-84,85$ plus), risk adjustment indicators, and ZIP3 dummies. We used the estimated coefficients $\widehat{\beta}_{t}^{E}$ and $\widehat{\beta}_{t}^{C}$ from this model to predict area-level expenditures and costs, holding constant the demographic and health characteristics of patients at their 1997 levels:

$$
\begin{aligned}
& \widehat{E}_{k t}=\bar{X}_{k, 1997} \widehat{\beta}_{t}^{E} \\
& \widehat{C}_{k t}=\bar{X}_{k, 1997} \widehat{\beta}_{t}^{C}
\end{aligned}
$$

We define generosity in zip code $k$ at time $t, G_{k t}$, as $G_{k t}=\hat{E}_{k t} / \hat{C}_{k t}$. Our SNF generosity measures were constructed in an analogous way. 


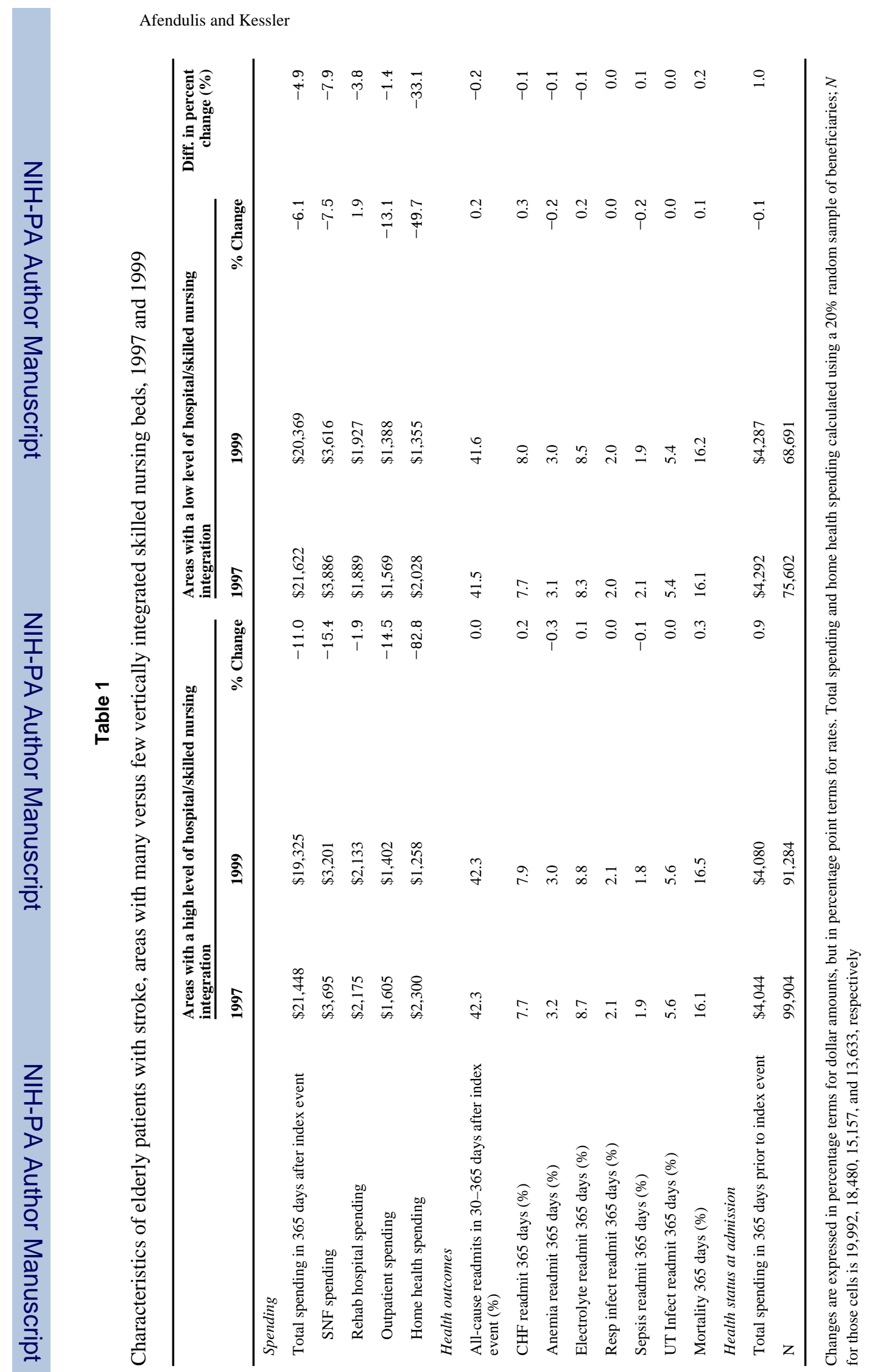

Page 12

Int J Health Care Finance Econ. Author manuscript; available in PMC 2011 October 17. 



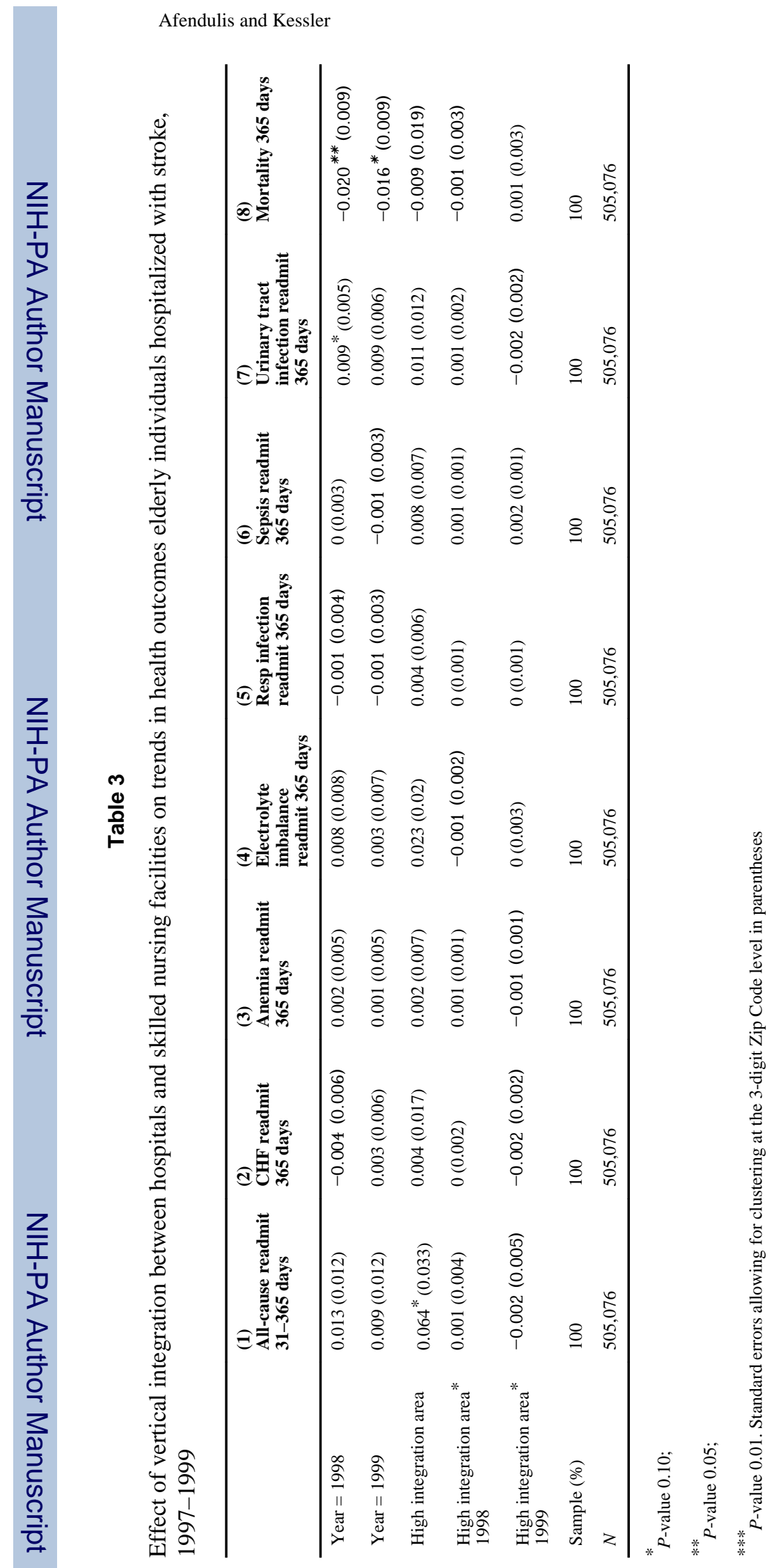

Int J Health Care Finance Econ. Author manuscript; available in PMC 2011 October 17. 